\title{
Two Kinds of Activity in Late-type Stars
}

\author{
M.M. Katsova \\ Sternberg State Astronomical Institute, \\ Moscow State University, 119899 Moscow V-234, USSR
}

Several years ago we proposed a method for the analysis of X-ray observations of late-type stars. It allowed the determination in a uniform manner of coronal base electron densities for more than 40 late-type stars, in terms of a one-temperature consideration of homogeneous spherically symmetric coronae (Katsova et al., 1987). Fig. 1 shows the results as a function of spectral type. Comparison of our results with values for different kinds of solar regions shows that physical characteristics of $\mathrm{F}$ and $\mathrm{G}$ star coronae correspond to densities less than those in active regions on the Sun. Values for the active K-M0 stars are comparable with those of dense steady condensations found directly above large sunspots.

On this basis, activity can be explained as an increase in that part of the stellar surface that is occupied by strong local magnetic fields. This is illustrated in the table where we compare magnetic field measurements by Saar and Linsky (1988) with our calculations.

Table

\begin{tabular}{ccccc}
\hline Star & Sp.class & H,G & $f$ & $n_{0} \cdot 10^{8} \mathrm{~cm}^{-3}$ \\
\hline Sun & dG2 & 1500 & $\sim 0.02$ & 4 \\
40 Eri A & dK1 & $2800 \pm 700$ & 0.07 & 2.2 \\
HD 17925 & dK2 & 1500 & 0.35 & 3.7 \\
PZ Mon & dK2e & $2200 \pm 200$ & $0.80 \pm 0.20$ & 5.6 \\
61 Cyg B & dK7 & 1500 & $<0.1$ & 11 \\
EQ Vir & dK5e & $2500 \pm 300$ & $0.80 \pm 0.15$ & 35 \\
AD Leo & dM4.5e & $3800 \pm 260$ & $0.73 \pm 0.06$ & 54 \\
AU Mic & dM2.5e & 4000 & 0.9 & 69 \\
\hline
\end{tabular}

When we go from $G$ to $M$ stars, the filling factor $f$ can reach 0.8. Apparently, we are dealing here with the enhancement of solar-like activity processes.

Vilhu (1987) showed that the X-ray flux increases when we consider later-type stars. The X-ray emission for stars with values of $F_{\mathrm{x}} / F_{\mathrm{bol}}<10^{-4}$ is connected with emission of coronal gas with $T \approx 2-3 \mathrm{MK}$. For stars with $F_{\mathrm{x}} / F_{\text {bol }} \geq 10^{-4}$ $10^{-3}$ and the same spectral type, the $\mathrm{X}$-ray luminosity begins to be determined by 
high-temperature gas with $T \approx 20 \mathrm{MK}$. Apparently, this conclusion is valid both for the K-stars that are the active components in RS CVn-binaries, and for the M-dwarf stars.

This is illustrated in Fig. 2, where the emission measure EM of low $\left(T_{1}\right)$ and high $\left(T_{2}\right)$ temperature gas is plotted for different stars, using EXOSAT data by Schmitt et al. (1987). For comparison, emission measures are given also for a solar active region and for the maximum of a solar flare.

We note that the slope of the lines that connect two-temperature component emission measures, differs from those in a solar active region for the case of the RS CVn-binary $\sigma^{2} \mathrm{CrB}$. Dwarf stars with the highest level of surface activity show similar behaviour. In these stars the hot gas component begins to dominate over the low-temperature component of the coronal plasma.

What causes the appearance of such hot gas in stellar coronae? Observations of RS CVn-type binary systems suggest the existence of interaction processes involving large stellar magnetic loops located on both stars. This may give rise to the development of flare-like process magnetic field reconnection. During this process the large scale loops are filled by hot plasma with $T=20 \mathrm{MK}$. Such flare loops have apparently been observed in the eclipsing binary AR Lac.

Two immediate questions remain for future research: Why are such flare loops long-lived? How are they enchanced during prolonged flares on these stars?

In stars of spectral class $M$, the red dwarf stars, we are faced with a different situation. These stars have the same ratio $F_{\mathrm{x}} / F_{\text {bol }} \approx 10^{-3}$ as the $\mathrm{K}$-stars. The $\mathrm{X}$-ray emission of hot plasma occurs continually. Their radii and luminosities are small, but nevertheless there is a high efficiency of X-ray generation. In contrast to the $\mathrm{K}$ components of the RS CVn-systems, large hot loops apparently are absent from the coronae of active red dwarf stars.

In the past few years microflaring has been discussed as a possible mechanism of heating red dwarf star coronae. Now additional arguments can be given in favour of this viewpoint. Firstly, variability of the Balmer line emission can be traced in spectroscopic observations with high temporal resolution, carried out on the 6-m telescope (Pustilnik, 1990). Secondly, the observations of the Balmer decrement in quiescent red dwarf stars, provided by Pettersen and Hawley (1989), show that stars with high $\mathrm{X}$-ray luminosities have larger fluxes in the $\mathrm{H} \alpha$-line, in contrast to stars without X-ray emission. Comparison of the Balmer decrement observations with theoretical results for different values of temperature, density and optical depth at the L $\alpha$-line centre (Katsova, 1990) shows that physical conditions in the emitting source are close to those found in the plasma of small flares.

Thus, we have certain evidence in favour of the existence of two types of stellar activity: i) a quasistationary process of enhancement of solar-like activity with increasing filling factor due to large-scale structure; ii) a nonstationary process of development of small-scale activity such as microflaring. 


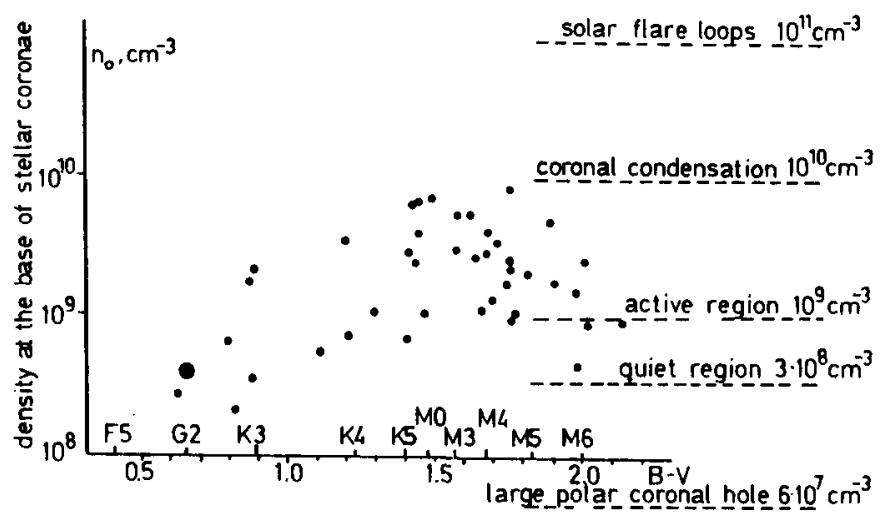

Fig. 1.

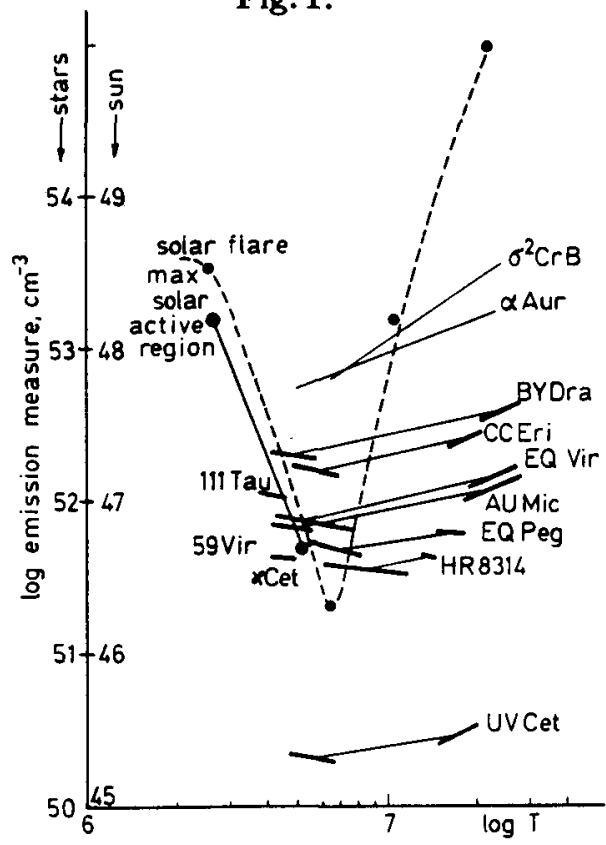

Fig. 2.

\section{References}

Katsova, M.M.: 1990, Sov. Astron., in press

Katsova, M.M., Badalyan, O.G., Livshits, M.A.: 1987, Sov. Astron. 31, 652

Pettersen, B.R., Hawley, S.L.: 1989, Astron. Astrophys. 217, 187

Pustilnik, L.A.: 1990, private communication

Saar, S.H., Linsky, J.L.: 1986, in Fourth Cambridge Workshop on Cool Stars, Stellar Systems and the Sun, Santa Fe, New Mexico, p. 93

Schmitt, J.H.M.M., Pallavicini, R., Monsignori-Fossi, B.C., Harnden, F.R., Jr.: 1987 Astron. Astrophys. 179, 193

Vilhu, O.: 1987, in Fifth Cambridge Workshop on Cool Stars, Stellar Systems and the Sun, Boulder, Colorado, p. 104 